\title{
Thermal Dose Control of Ultrasound Therapies using MR Thermometry Images: An In-Vitro Phantom Study
}

\author{
Dhiraj Arora, Daniel Cooley, Trent Perry, Junyu Guo, Dennis Parker, Mikhail Skliar and Robert Roemer
}

\begin{abstract}
An MR-based thermal treatment controller that delivers a specified thermal dose to a selected target has been developed and evaluated in-vitro using a magnetic resonance (MR) compatible focused ultrasound heating system. The thermal treatment control system has a cascade structure with the main nonlinear dose controller continuously generating the reference temperature trajectory for the secondary constrained, model predictive temperature controller. The control system allows the physician to impose constraints on the maximum allowable temperature elevation at the selected normal tissue location. To reflect hardware imitations and to prevent tissue cavitation, constraint on the maximum transducer power can also be imposed. The combination of a surface coil designed for in-vitro experiments, a gradient echo sequence with $\boldsymbol{k}$-space reduction and a 3T scanner allowed for fast $(1.45 \mathrm{~s})$ and low noise $\left( \pm 0.5^{\circ} \mathrm{C}\right)$ MR temperature acquisition. The noninvasive thermal images are used during the pre-treatment heating session to characterize the spatial distribution of applied power and effective perfusion, and for the online feedback control of target thermal dose. During the in-vitro phantom experiments the prescribed thermal dose was delivered to the target while restricting the temperature elevation at a selected normal tissue location to below a specified limit. The results demonstrate the robustness of the developed MR-based thermal dose controller in terms of target dose delivery and normal tissue safety.
\end{abstract}

\section{INTRODUCTION}

The potential of focused ultrasound thermal therapies for tumor treatment of various anatomical sites such as brain, prostate and breast has been demonstrated by a number of successful clinical trials [1], [2], [3] [4]. Positive results of ultrasound therapies have been reported for tissue ablation and necrosis [1], [2], and as an adjuvant modality in conjunction with radiation [3] and chemotherapy [4]. From the clinical perspective, the goal of the ultrasound treatments is to achieve a desired thermal dose (TD) distribution in the tumor while maintaining temperature elevation below a safe level in the healthy tissue to minimize patient pain, discomfort and potential damage.

In the current clinical setting, in order to avoid damage to the healthy tissue, the clinician often adopts a conservative

This work was supported in part by NIH grant NIH-1-ROI-CA87785 and NSF grant CTS 0117300 .

D. Arora, D. Cooley, T. Perry and R. Roemer are with the Department of Mechanical Engineering, University of Utah.

J. Guo and D. Parker are with the Utah Center for Advanced Imaging Research (UCAIR), University of Utah, Salt Lake City, Utah.

M. Skliar is with the Department of Chemical Engineering, University

of Utah. Send correspondence to mikhail.skliar@utah. edu treatment protocol by limiting the maximum ultrasound power or using pulsed heating (i.e. heating separated by cool-down periods [5]). It is advantageous to replace treatment based on clinician's intuition and experience with a thermal dose controller that delivers the desired thermal dose to the target in the presence of disturbances such as changing blood flow [6] and ultrasound absorption [7]. Furthermore, the clinician-driven conservative approach may lead to prolonged treatment and increased cost. Therefore, there exists a need for an automatic control system that delivers the prescribed thermal dose to the target in the minimum-time without causing normal tissue damage.

A number of thermal therapy controllers designed to control temperature(s) in selected spatial locations [8], [9], have been proposed in recent years. For instance, in is demonstrated in [8] that temperature control in a plane based on MR thermometry and spiral path scanning of an ultrasound focal zone. In the simulation study [9], the feasibility of a MR-thermometry based LQR controller to achieve a desired temperature-time profile in a single point of interest was demonstrated. Our earlier simulation work [10] showed that in the presence of unmeasured disturbances, controlling temperatures, as attempted in the past, may cause significant error in the delivered thermal dose.

The thermal treatment control problem was previously formalized as the problem of direct, time-optimal feedback control of the thermal dose delivered to the tumor subject to constraints on the maximum allowable normal tissue temperature. The detailed description of the thermal dose controller that solves the posed problem is reported in [10], where the controller performance was evaluated using computer simulations. The developed model-based thermal dose controller was shown to achieve a near time-optimal treatment in the presence of substantial modeling errors. It also allows for feedback in the form of MR thermal images. An advantage of the MR-compatible controller is the ability to implement a noninvasive thermal dose controller based on comprehensive intra-treatment temperature measurements.

This paper presents a successful in-vitro evaluation of our previously developed thermal dose controller [10] with the incorporation of MR thermometry feedback. The MRbased controller results show that a desired thermal dose is robustly delivered to the target in a time optimal manner while limiting the peak temperature in a selected location 
adjacent to the target below a specified value.

\section{METHODS}

The following describes the temperature response and thermal dose models, model-based TD controller, experimental setup, MR thermometry, and identification procedure for obtaining specific absorption rate (SAR) and blood perfusion models.

\section{A. Temperature and thermal dose models}

The internal model used by the controller to predict the temperature response in the phantom was assumed to obey a 1-dimensional fin heat transfer equation:

$$
\rho C \frac{\partial T}{\partial t}=k \frac{\partial^{2} T}{\partial x^{2}}-W_{e} C_{b}\left(T-T_{a}\right)+Q
$$

This temperature response model is similar to Pennes' bioheat transfer equation [11] traditionally used to characterize temperature response in the tissue. The parameter $W_{e}$, which in the Pennes' model represents the effect of blood perfusion, in equation (1) accounts for the energy removed by conduction in the planes perpendicular to the ultrasound axis in the assumed one-dimensional model. Parameters $C$ and $C_{b}$ are the specific heat of tissue and blood $[\mathrm{J} /(\mathrm{kg}$ $\left.{ }^{\circ} \mathrm{C}\right)$ ]. $T_{a}$ is the ambient temperature, which was assumed to be constant for the duration of the experiments. The baseline temperature was set equal to $T_{a}$ and the results are reported as the deviation of the phantom's temperature from the baseline. $Q\left[\mathrm{~W} / \mathrm{m}^{3}\right]$ is the power deposition density, also known as SAR. At the boundaries of the domain, $T=T_{a}$ is assumed. Handbook values for conductivity $k=0.7 \mathrm{~W} /\left(\mathrm{m}^{\circ} \mathrm{C}\right)$ and the specific heat $C=C_{b}=4186$ $\mathrm{J} /\left(\mathrm{kg}^{\circ} \mathrm{C}\right)$ were assumed.

The one-dimensional approximation (1) was made to reduce on-line computational requirements. The values of effective perfusion parameter and the SAR were identified experimentally as described later in the paper.

The internal model used in the constrained model predictive controller is the finite difference approximation of the spatially distributed model (1) expressed in the state space form:

$$
\begin{gathered}
\dot{T}=A T+B u \\
T_{m e a}=C T
\end{gathered}
$$

where $A$ is the system matrix that depends on both conduction and perfusion. The term $B u$ approximates the power deposition term, where $u$ is the applied ultrasound power in Watts. The state $T$ is a vector of deviation temperatures above $T_{a}$ along the one-dimensional treatment domain. The position of the ultrasound transducer was fixed and the magnitude of the applied ultrasound power $u$ is the only manipulated variable. Since MR-based temperatures are available in each voxel of the treatment domain, the output matrix $C$ is an identity matrix.

The temperature evolution over time, $T(x, t)$, is converted into thermal dose, the commonly used thermal treatment

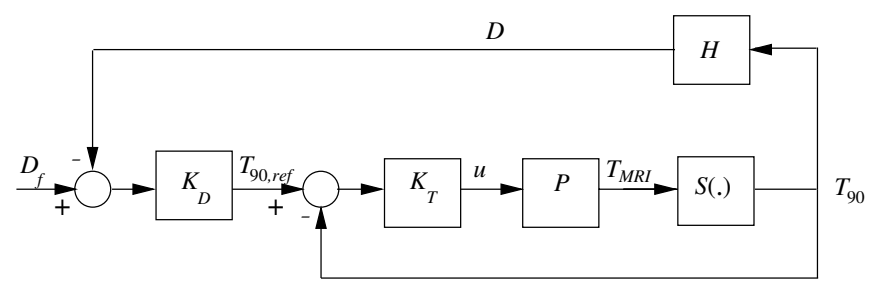

Fig. 1. Feedback control of the thermal dose; $K_{D}$ : Dose controller; $K_{T}$ : Temperature controller; $P$ : Patient; $S():. T_{90}$ selector and $H$ : Dose Estimator.

efficacy measure [12], calculated as cumulative equivalent minutes $(\mathrm{CEM})$ at $43^{\circ} \mathrm{C}$ :

$$
D(t)=\mathrm{CEM} \text { at } 43^{\circ} \mathrm{C} T_{90}=\int_{0}^{t} R^{\left[43-T_{90}(\tau)\right]} d \tau
$$

where $T_{90}$ is the $10^{\text {th }}$ percentile of the measured temperatures, and, $R=0$ for $T_{90}<2, R=0.25$ for $2<T_{90}<6$ and $R=0.5$ for $T_{90} \geq 6$.

\section{B. Thermal Dose Controller}

The controller is implemented as a cascade structure depicted in Figure 1 with the main nonlinear dose controller continuously generating a reference temperature trajectory for the secondary, constrained, model predictive temperature controller. A detailed description of this controller can be found in Arora et al 2004 [10], which also describes the results of the controller evaluation using computer simulations. The outer loop thermal dose controller, $K_{D}$, maps the difference between the desired final dose, $D_{f}$, and the current delivered thermal dose, $D\left(t_{k}\right)$, into the reference $10^{\text {th }}$ percentile temperature trajectory, $T_{90, \text { ref }}(t)$ :

$$
K_{D}:\left[D_{f}-D\left(t_{k}\right)\right] \rightarrow T_{90, r e f}(t), t \in\left[t_{k}, t_{f}\right]
$$

The map (5) is implemented using two inverse operators $\mathcal{M}_{1}$ and $\mathcal{M}_{2}$ :

$$
K_{D}\left[D_{f}-D\left(t_{k}\right)\right]=\mathcal{M}_{2}\left[\mathcal{M}_{1}\left(D_{f}-D\left(t_{k}\right)\right)\right]=T_{90, r e f}(t)
$$

where $\mathcal{M}_{1}$ maps the remaining dose deficit, $D_{f}-D\left(t_{k}\right)$, at each sampling instant into a reference dose trajectory $D_{\text {ref }}(t)$, and $\mathcal{M}_{2}$ maps $D_{\text {ref }}(t)$ into the reference $T_{90, r e f}(t)$. Since there are infinitely many reference dose (and temperature) trajectories that will result in the same final desired dose, $D_{f}$, the uniqueness of the map $\mathcal{M}_{1}$ is ensured by using the following linear parametrization of the $D_{\text {ref }}(t)$ :

$$
D_{\text {ref }}(t)=D\left(t_{k}\right)+\alpha\left(t_{k}\right)\left[t-t_{k}\right]
$$

The slope $\alpha$ is calculated based on the difference between the delivered and the desired thermal dose, and the selected final treatment time:

$$
\alpha\left(t_{k}\right)=\frac{\left(D_{f}-D\left(t_{k}\right)\right)}{\left(t_{f}-t_{k}\right)}
$$


where $t_{f}=t_{k}+\Delta t$, and the tuning parameter $\Delta t$ is the moving treatment horizon.

With the obtained $D_{r e f}, \mathcal{M}_{2}$ is simply the inverse of the integral equation (4), resulting in the following reference temperature trajectory:

$$
T_{90, \text { ref }}(t)=\frac{1}{\ln (1 / R)} \ln \frac{\alpha\left(t_{k}\right)}{R^{43}}, \quad t \in\left[t_{k}, t_{k}+\Delta t\right]
$$

which is updated each time a new thermal dose estimation becomes available. The developed primary controller $K_{D}$ is a particular case of the generic model controller (GMC) [13], which involves implicit inversion of a (possibly nonlinear) process model.

To allow imposition of a constraints on the maximum allowable temperature at the selected spatial location of the normal tissue and transducer saturation, $K_{T}$ is implemented as a constrained, linear model predictive controller. The predictive model used in the design of $K_{T}$ is given by equations 2 and 3 . The optimizing controller $K_{T}$ generates the $m$ future control moves, $\mathbf{u}=[u(k), \ldots, u(k+m-1)]$, as a solution to the following optimization problem:

$$
\begin{aligned}
J(k) & =\sum_{j=1}^{p} w_{y}(j)\left[T_{90, \text { ref }}(k+j)-T_{90}(k+j)\right]^{2} \\
& +\sum_{j=1}^{m} w_{u}(j)[u(k+j-1)]^{2}
\end{aligned}
$$

subject to the process model (2)-(3), and temperature and actuation constraints:

$$
\begin{array}{lc}
T_{n}(k+j) \leq T_{n, \max } & j \in[1, p] \\
0 \leq u(k+j-1) \leq u_{\max } & j \in[1, m]
\end{array}
$$

where $w_{y}$ is the penalty on the error between the desired and the estimated $T_{90}, w_{u}$ is the penalty on the control effort, $T_{n, \max }$ is the maximum allowable temperature at the selected normal tissue location, $x_{n}$, and $u_{\max }$ is the transducer saturation limit. The prediction horizon $p$ determines the time over which the temperature predictions are made. The prediction horizon is selected to be long enough to estimate the thermal dose accrued during tissue cooling after power is turned off. The control horizon $m \leq p$ determines the number of future control moves that are calculated each time a new measurement becomes available. Only the first move, $u(k)$, is actually applied to the patient, and the procedure is repeated at the next sampling instant. The effect of the tuning parameters on the controller performance is detailed in our earlier work [10].

\section{Temperature Measurements}

MR imaging was performed using a Siemens Trio three Tesla Magnetom scanner. The temperature changes in the agar phantom were measured using the proton resonance frequency (PRF) shift method [14]. Phase difference image data were gathered using a gradient echo (GRE) pulse

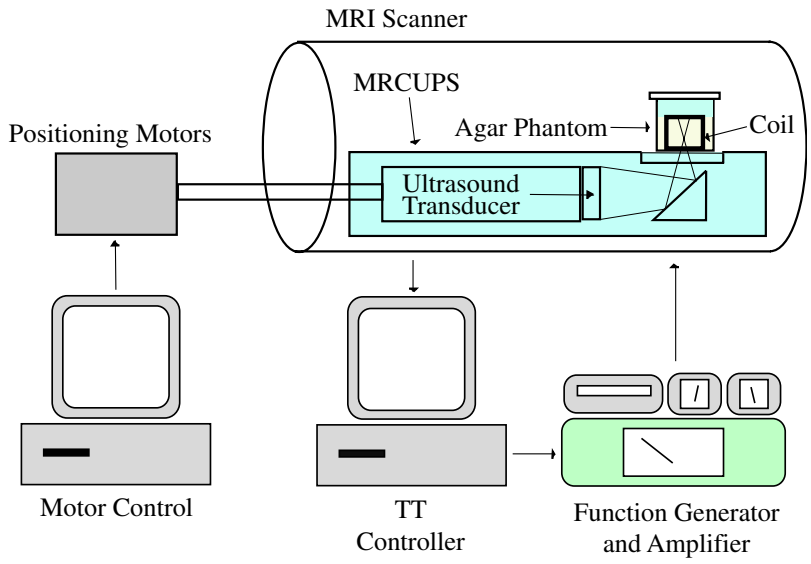

Fig. 2. Treatment control system and the phantom positioned inside the MRI bore.

sequence with a spoiled gradient. The following parameters were used: $\mathrm{TR}=30, \mathrm{TE}=10$, voxel size $=2.0 \times 4.0 \mathrm{x}$ $3.0 \mathrm{~mm}, \mathrm{FOV}=300 \mathrm{~mm}$, matrix $=128 \times 64$, flip angle $=$ 25 , scan time $=1.15$ or $2.6 \mathrm{~s}$ with a phase resolution of fifty percent to reduce the scan time. The $k$-space data were zerofilled to a $128 \times 128$ matrix. The temperature change in the agar phantom was calculated using the following equation:

$$
\Delta T=\frac{\Delta \phi}{\gamma \cdot \alpha \cdot B_{o} \cdot T E}
$$

where $\Delta \phi$ is the phase difference, $\gamma$ is the gyromagnetic ratio, $\alpha$ is the temperature coefficient (0.01ppm [14]), $B_{o}$ is the main field strength, and $T E$ is the time to echo.

\section{Experimental Setup and Procedures}

The control experiments were performed using a $11 \times 11$ $x 7 \mathrm{~cm}$ agarose phantom. One mol/liter of copper sulfate was added to the phantom to provide the desired $\mathrm{T} 2$ relaxation. The tissue mimicking material (agar with condensed milk) was contained inside an acrylic box with a thin mylar membrane on the bottom surface. Prior to the experiment, a custom-built receive-only surface coil was attached to the side of the phantom and was tuned and matched for optimal signal-to-noise ratio. The coil tuning ad matching were performed using a Hewlett Packard, Inc. spectrum analyzer, Model 8752A.

An in-house manufactured MR-compatible ultrasound positioning system (MRCUPS) was used to mechanically steer the ultrasound beam. The ultrasound field was generated by a single, spherically focused, air-backed transducer resonating at $1.5 \mathrm{MHz}$ with a diameter and radius of curvature of 10 and $18 \mathrm{~cm}$, respectively. The ultrasound beam was reflected off a 45 degree mirror to a mylar treatment window at the top of the acrylic chamber (Figure 2 ). The chamber of the positioning system was filled with deionized and degassed water.

The phantom was positioned on MRCUPS in the center of the Mylar treatment window with the coil in the saggital plane. Ultrasound gel was used to couple the phantom 


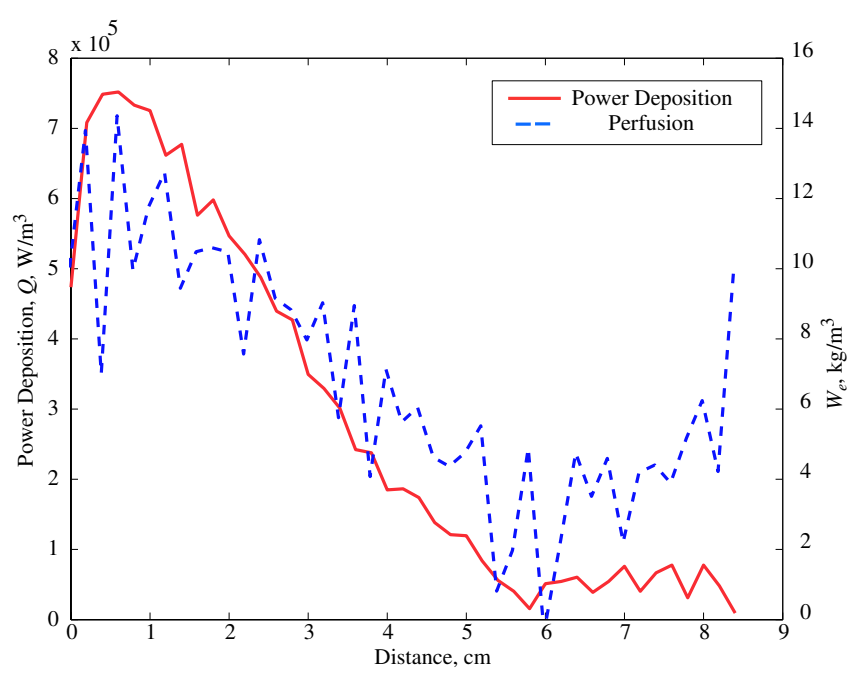

Fig. 3. Pre-treatment parameter identification: Power deposition pattern, $Q(x)$, and perfusion, $W_{e}(x)$.

to the Mylar window. To locate the focal zone, a step input of ultrasound power was applied to the phantom while phase images were acquired approximately halfway through the phantom. Based on the MR-thermal image, a saggital slice corresponding to maximum temperature was chosen through the center of the heated region, and was subsequently used as the treatment domain of all control experiments.

\section{E. Parameter Identification}

The perfusion related parameter, $W_{e}(x)$, and the SAR or power deposition density, $Q(x)$, are needed to implement the patient- and site-specific model predictive temperature controller $K_{T}$. These parameters were identified from the temperature response recorded by the MRI to 5 and $7 \mathrm{~W}$ step increases in applied ultrasound power during a pretreatment heating session. For these identification tests, MR-based temperature measurements were acquired once every $2.45 \mathrm{~s}$. Since conduction and convection effects are negligible immediately after the power is initiated [15], equation (1) simplifies to:

$$
\rho C \frac{\partial T(x)}{\partial t}=Q(x)
$$

Therefore, $Q$ can be estimated at each voxel from the initial slope of the recorded temperatures.

The effective perfusion, $W_{e}(x)$, was obtained from the measurements of the steady state temperatures reached in response to the step change in ultrasound power. At the steady state, equation (1) reduces to

$$
k \frac{\partial^{2} T}{\partial x^{2}}-W_{e}(x) C\left[T-T_{a}\right]+Q(x)=0
$$

from which spatial distribution of $W_{e}$ was identified by solving the least squares problem of minimizing the difference between experimental and model-predicted steady state temperatures. The iterations of the least squares fitting
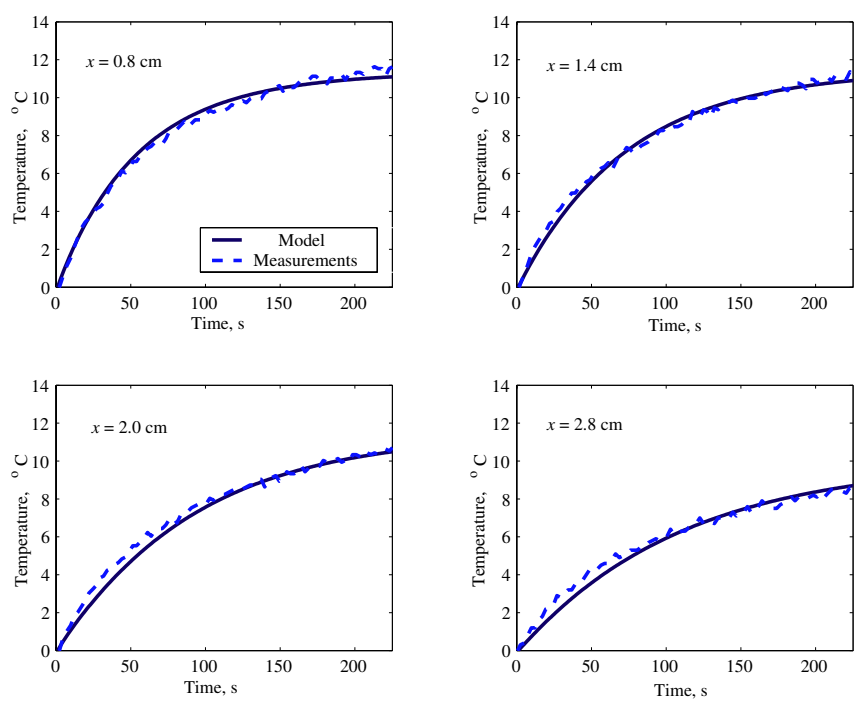

Fig. 4. Comparison of model predicted and measured temperature at four spatial locations in the phantom.

algorithm are stopped when the improvement between the successive steps is less than $0.1 \%$.

The identified $W_{e}(x)$ and $Q(x)$ for the agar phantom are depicted in Figure 3. The obtained power deposition density, $Q(x)$, is for the power step of $u=7 \mathrm{~W}$. In the phantom, the region between $x=0.4$ and $x=2.2 \mathrm{~cm}$ was selected as the "tumor". The maximum allowable normal tissue constraint was imposed in the proximity of the tumor at $x=2.8 \mathrm{~cm}$.

Figure 4 shows the comparison of the MR measured and model predicted temperature change in response to the step increase in the transducer power $(u=7 \mathrm{~W})$. The figure depicts temperature comparison at four locations, three inside the "tumor" and one at the selected normal tissue constraint location. The comparison shows a good agreement between the measured temperatures and the temperatures predicted by the one-dimensional thermal model (1).

\section{RESULTS}

The performance of the proposed thermal dose controller was evaluated by conducting several sets of in-vitro experiments with different maximum power levels $\left(u_{\max }\right)$, values of tuning parameters and normal tissue constraint $\left(T_{n, \max }\right)$. The effect of the tuning parameters and the value of the normal tissue constraint on the controller performance was qualitatively similar to our previous simulation [10] results. In this paper, two sets of results with different allowable power levels are presented.

The objective of the performed experiments was to deliver a desired thermal dose to the selected target region while limiting the temperature at the constraint location to below $6.5^{\circ} \mathrm{C}$. A setpoint dose of $10 \mathrm{CEM} 43^{\circ} T_{90}$ was chosen to reduce the cost of MR time. Earlier phantom experiments [16] using thermocouple array have clearly shown the ability of the thermal dose controller to deliver higher values of desired dose. To reflect hardware constraints, an upper 

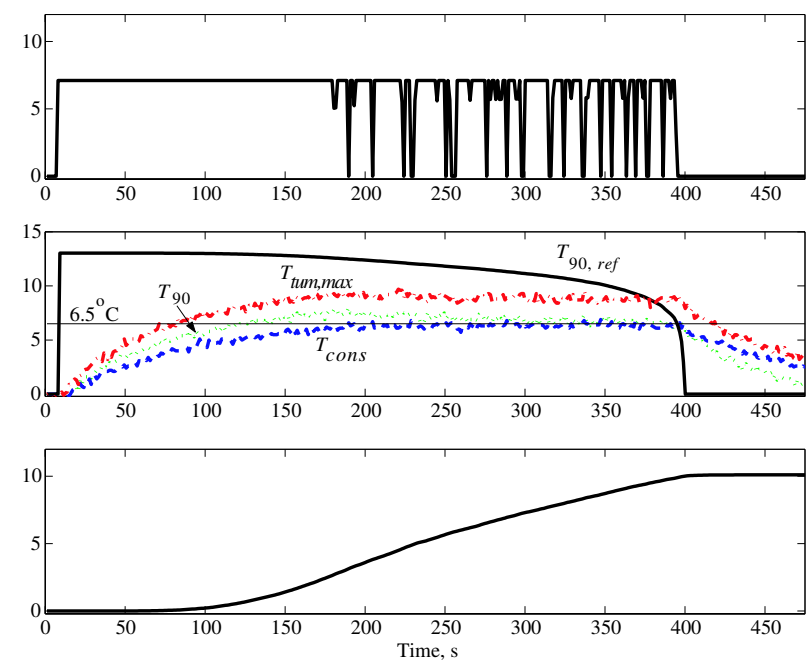

Fig. 5. Thermal dose control with $u_{\max }=7 \mathrm{~W}$. The desired CEM $43^{\circ}$ $T_{90}$ was set to $10 \mathrm{~min}$. The maximum allowable temperature increase at the specified location inside the normal tissue was $6.5^{\circ} \mathrm{C}$. The results were obtained with the moving treatment horizon $\Delta t=4.6$, prediction horizon $p=4.6$, and the control horizon $m=2.3$. (a) Control input. (b) Increase in $T_{90}, T_{90, \text { ref }}, T_{\text {cons }}$ and $T_{\text {tum,max }}$. (c) Tumor thermal dose

level constraint on applied ultrasound power, $u_{\max }$, was used during the controller experiments.

The first set of results was obtained with the ultrasound power constrained to $u_{\max }=7 \mathrm{~W}$. Figure 5 depicts the evolution of the controller output power, $u$, maximum target temperature, $T_{t u m, \max }, T_{90}$ in the target, temperature at the constraint location, $T_{\text {cons }}$, the reference temperature generated by the outer controller, $T_{90, \text { ref }}$, and the thermal dose in the target. The controller tuning parameters $p$ and $m$ were set to 4.6 and $2.3 \mathrm{~s}$, respectively. The value of the moving treatment horizon, $\Delta t=4.6 \mathrm{~s}$, was selected to force the activation of the transducer constraint at the beginning of the treatment when the normal tissue temperature constraint was not active. At $t=180 \mathrm{~s}$, Figure 5(b), the temperature constraint became active. The constrained model predictive controller, $K_{T}$, reduced the ultrasound power, Figure 5(a), in such a way that the temperature at the constraint location is maintained near the specified value of $6.5^{\circ} \mathrm{C}$. The desired thermal dose of $10 \mathrm{CEM} 43^{\circ} T_{90}$ was achieved in the target at approximately $t=405 \mathrm{~s}$.

In the second experiment, the maximum allowable transducer power was raised to $u_{\max }=11 \mathrm{~W}$ to investigate the effect of a higher applied power on the treatment time. The results are shown in Figure 6. The controller tuning parameters $p, m$ and $\Delta t$ were the same as in the previous experiments. With a higher allowable transducer power, the normal tissue constraint became active after only 103s. As a result, the treatment time was considerably shorter than in the first experiment, taking only 340 s to reach $10 \mathrm{CEM} 43^{\circ} T_{90}$. Similar to the previous case, the controller actively modulates the power, Figure 6(a) to ensure that the temperature in the constraint location is maintained near the specified peak value of $6.5^{\circ} \mathrm{C}$.
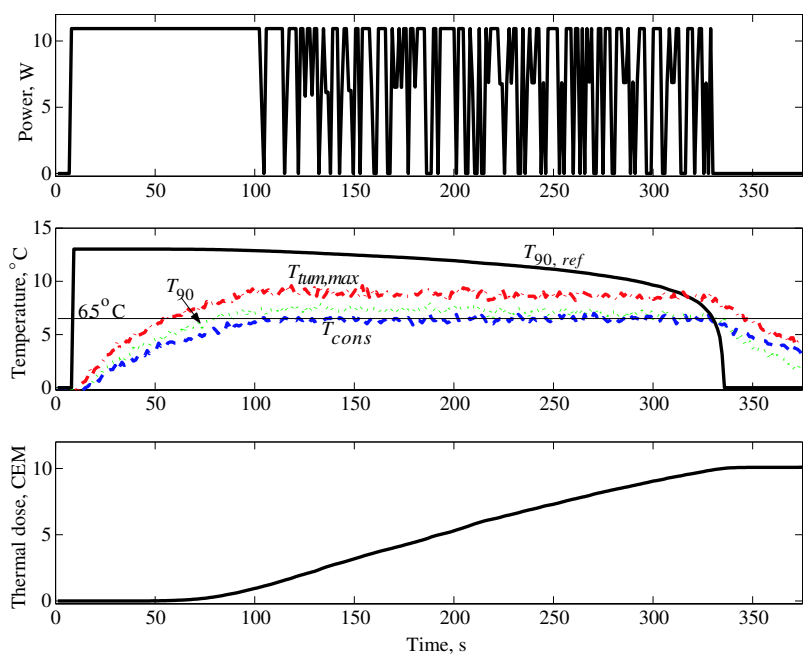

Fig. 6. Thermal dose control with $u_{\max }=11 \mathrm{~W}$. The desired CEM43 ${ }^{\circ}$ $T_{90}$ was set to $10 \mathrm{~min}$. The maximum allowable temperature increase at the specified normal tissue location was $6.5^{\circ} \mathrm{C}$. The results were obtained with the moving treatment horizon $\Delta t=4.6$, prediction horizon $p=4.6$, and the control horizon $m=2.3$. (a) Control input. (b) Increase in $T_{90}$, $T_{90, \text { ref }}, T_{\text {cons }}$ and $T_{\text {tum,max }}$. (c) Tumor thermal dose

\section{DISCUSSION}

The presented in-vitro results demonstrate the successful validation of a MR-based thermal dose controller, which meets the objective of delivering a desired thermal dose to the target in a time optimal manner while automatically restricting the normal tissue temperature to below a specified value.

Direct thermal dose feedback control can replace the need for trial-and-error manual changes by a clinician attempting to deliver the desired thermal dose. The capability of thermal dose control to deliver a specified thermal dose is enhanced by the use of MR thermometry that provides spatially distributed measurements of temperatures, reducing the uncertainty compared to the case when sparse, pointwise and invasive temperatures measurements using thermocouple array or fiberoptic temperature probes are used.

The proposed controller delivers the thermal dose to the target in a time-optimal manner without damaging the normal tissue. Trial-and-error estimates of the ultrasound pulsing in order to ensure normal tissue safety often result in an overly conservative approach, which leads to a significantly longer treatment times. On the other hand, an $a d$ hoc selection of too high of a power level or long power-on cycles will lead to excessive normal tissue heating characterized by patient pain, discomfort and potential normal tissue damage. The proposed near time-optimal TD controller allows the clinician to explicitly specify the desired target thermal dose and the normal tissue constraint, thus removing the need for the trial-and-error selection of the ultrasound actuation.

The results of Figures 5(a) and 6(a) show that active switching in ultrasound power occurs in order to maintain 
the temperature at the constraint normal tissue location close to the specified maximum allowable value. The Pontryagin's maximum principle [17] requires that the treatment must progress with either the normal tissue temperature or maximum power constraint active in order to achieve the minimum time treatment. The observed oscillatory behavior is typical of aggressive (such as a minimum-time) and constrained controllers when applied to processes affected by time-varying disturbances and in the presence of modeling errors. The earlier simulation results [10] showed that in the ideal case of no plant-model mismatch, and with a time-invariant disturbance, the controller arrives at an exact power level to maintain the normal tissue at the constrained value, completely eliminating the active modulation of the ultrasound power. If desired, the rapid change in power can be reduced by using a larger value of the tuning parameter $w_{u}$ in objective function (10). However, this change will generally lead to a longer treatment. In order to obtain a near minimum time results presented in this paper, $w_{u}$ was set to zero.

The controller tuning used to obtain the presented invitro results was based on the experience and guidelines obtained during earlier simulation [10] and in-vitro phantom studies with thermocouple measurements [16]. The guidelines suggest the procedure to select the moving treatment horizon $\Delta t$, which leads to a near time-optimal treatment without explicitly solving in real time a computationally intensive minimum-time optimal control problem. Based on the experimental results of multiple studies, including those presented in the current paper, the effect of controller tuning is in qualitative agreement with the expectations, thus validating the developed guidelines.

The controller presented in this paper allows specification of the normal tissue constraints in terms of the maximum temperature and not the thermal dose. Although attractive because of a potential reduction in treatment time, adopting the approach of imposing explicit constraints on maximum allowable normal tissue thermal dose generally leads to a non-causal control problem, as discussed in more detail in our earlier work [10].

\section{CONCLUSIONS AND FUTURE WORKS}

The developed and implemented MR-based TD controller provides several significant clinical advantages such as the direct, feedback control of thermal dose, explicit constraint inclusion to limit the damage in the normal tissue and the ability to achieve time-optimal treatments. The direct thermal dose feedback controller robustly delivers the desired TD to the target in a time optimal manner while maintaining the normal tissue temperature below a specified value.

As part of our ongoing and future work, we are developing treatment controllers with MR thermometry feedback that use scanning focal zones, obtained using mechanical motion of a single focused transducer, or electronic shaping of the SAR pattern with ultrasound phased arrays.

\section{ACKNOWLEDGMENTS}

The authors wish to thank Andrew Richardson and Jeff Moellmer (both, School of Computing, University of Utah) for developing the software to interface MR imaging and thermal treatment computers, and implementing the graphical user interface to monitor treatment progression.

\section{REFERENCES}

[1] K. Hynynen, O. Pomeroy, D.N. Smith, P.E. Huber, N.J. McDannold, J.K. Kettenbach, J.Baum, S. Singer, and F.A. Jolesz. MR imagingguided focused ultrasound surgery of fibroadenomas in the breast: A feasibility study. Radiology, 219:176-185, 2001.

[2] F. Wu, W.Z. Chen, J. Bai, Z.L. Wang, H.Zhu, and Z.B. Wang. Pathological changes in human malignant carcinoma treated with high-intensity focused ultrasound. Ultrasound Med. Biol., 27:10991106, 2001.

[3] A.N. Guthkelch, L.P. Carter, J.R. Cassady, K.H. Hynynen, R.P. Iacono, P.C. Johnson, E.A. Obbens, R.B. Roemer, J.F. Seeger, and D.S. Shimm. Treatment of malignant brain tumors with focused ultrasound hyperthermia and radiation: results of phase i trial. $J$. Neuro Oncol., 10:271-284, 1991.

[4] B.A. Bornstein, P.S. Zouranjian, J.L. Hansen, S.M. Fraser, L.A. Gelwan, B.A. Teicher, and G.K. Svensson. Local hyperthermia radiation therapy and chemotherapy in patients with local regional recurrence of breast carcinoma. Int. J Radiation Oncol Biol Phys., 25:79-85, 1993.

[5] A.L. Malcom and G.R. ter Haar. Ablation of tissue volumes using high intensity focused ultrasound. Ultrasound Med. Biol., 22:659669, 2001.

[6] D.P. Anhalt, K. Hynynen, and R. B. Roemer. Patterns of change of tumour temperatures during clinical hyperthermia: Implications for treatment planning, evaluation and control. Int. J. Hyperthermia, 11:425-436, 1995.

[7] F. Dunn and J.K. Brady. Absorption of ultrasound in biological media. Biophysics, 18:1128-1132, 1973.

[8] R. Salomir et al. Local hyperthermia with MR-guided focused ultrasound: Spiral trajectory of the focal point optimized for temperature uniformity in the target region. J. Mag. Res. Imaging, 12:571-583, 2000.

[9] A Vanne and K Hynynen. MRI feedback temperature control for focused ultrasound surgery. Phys. Med. Biol., 48:31-43, 2003.

[10] D. Arora, M. Skliar, and R. B. Roemer. Minimum time control of thermal therapies. IEEE Trans. Biomed. Eng., 52:191-200, 2005.

[11] H.H. Pennes. Analysis of tissue and arterial blood temperatures in resting human forearm. J. Appl. Physiol., 1:93-122, 1948.

[12] S.A. Sapareto and W.C Dewey. Thermal dose determination in cancer therapy. Int. J. Oncology Biol. Phys., 10:787-800, 1984.

[13] P.L. Lee and G.R. Sullivan. Generic model control (GMC). Computers and Chemical Engineering, 12:573-580, 1989.

[14] A.H. Chung, K. Hynynen, H.E Cline, V. Colucci, K. Oshio, and F. Jolesz. Optimization of spoiled-gradient-echo phase imaging for in-vivo location of focused ultrasound beam. Magn. Reson. Med., 36:745-752, 1996.

[15] R.B. Roemer, A.M. Fletcher, and T.C. Cetas. Local SAR and blood perfusion data from temperature measurements: Steady state and transient techniques compared. Int. J Radiation, 11:1539-1550, 1985.

[16] D. Arora, M. Skliar, D. Cooley, A. Blankespoor, J. Moellmer, and R. B. Roemer. Nonlinear model predictive thermal dose control of thermal therapies: Experimental validation with 1-D phantoms. In Proc. 2004 American Control Conference, pages 1627-1632, Boston, MA, June 2004.

[17] L.S. Pontryagin, V.G. Boltyanskii, R.V. Gamkrelidze, and E.F. Mishchenko. The Mathematical Theory of Optimal Processes. Wiley, New York, 1962. 